\title{
A propensity score analysis of the impact of surgical intervention on unexpected 30-day readmission following admission for subdural hematoma
}

\author{
Lynze R. Franko, BS, ${ }^{1}$ Kyle M. Sheehan, MD, ${ }^{2,3}$ Christopher D. Roark, MD, ${ }^{4}$ Jacob R. Joseph, MD, ${ }^{2}$ \\ James F. Burke, MD, ${ }^{3}$ Venkatakrishna Rajajee, MD, ${ }^{2,3}$ and Craig A. Williamson, MD $^{2,3}$ \\ ${ }^{1}$ University of Michigan Medical School; Departments of ${ }^{2}$ Neurosurgery and ${ }^{3}$ Neurology, University of Michigan, Ann Arbor, \\ Michigan; and ${ }^{4}$ Department of Neurosurgery, University of Colorado, Denver, Colorado
}

OBJECTIVE Subdural hematoma (SDH) is a common disease that is increasingly being managed nonoperatively. The all-cause readmission rate for SDH has not previously been described. This study seeks to describe the incidence of unexpected 30-day readmission in a cohort of patients admitted to an academic neurosurgical center. Additionally, the relationship between operative management, clinical outcome, and unexpected readmission is explored.

METHODS This is an observational study of 200 consecutive adult patients with SDH admitted to the neurosurgical ICU of an academic medical center. Demographic information, clinical characteristics, and treatment strategies were compared between readmitted and nonreadmitted patients. Multivariable logistic regression, weighted by the inverse probability of receiving surgery using propensity scores, was used to evaluate the association between operative management and unexpected readmission.

RESULTS Of 200 total patients, $18(9 \%)$ died during hospitalization and were not included in the analysis. Overall, 48 patients $(26 \%)$ were unexpectedly readmitted within 30 days. Sixteen patients (33.3\%) underwent SDH evacuation during their readmission. Factors significantly associated with unexpected readmission were nonoperative management $(72.9 \%$ vs $54.5 \%, p=0.03)$ and female sex $(50.0 \%$ vs $32.1 \%, p=0.03)$. In logistic regression analysis weighted by the inverse probability of treatment and including likely confounders, surgical management was not associated with likelihood of a good outcome at hospital discharge, but was associated with significantly reduced odds of unexpected readmission (OR 0.19, 95\% Cl 0.08-0.49).

CONCLUSIONS Over $25 \%$ of SDH patients admitted to an academic neurosurgical ICU were unexpectedly readmitted within 30 days. Nonoperative management does not affect outcome at hospital discharge but is significantly associated with readmission, even when accounting for the probability of treatment by propensity score weighted logistic regression. Additional research is needed to validate these results and to further characterize the impact of nonoperative management on long-term costs and clinical outcomes.

https://thejns.org/doi/abs/10.3171/2017.6.JNS17188

KEY WORDS patient readmission; intracranial subdural hematoma; chronic subdural hematoma; acute subdural hematoma; traumatic subdural hematoma; trauma

$\mathrm{U}$ NEXPECTED readmission following hospital discharge is common and costly, particularly in the elderly. Approximately $20 \%$ of Medicare patients are readmitted within 30 days, and unplanned hospitalizations were estimated to cost Medicare $\$ 17.4$ billion in $2004 .{ }^{15}$ More importantly, unexpected 30-day readmission is an independent predictor of increased mortality. ${ }^{10,11,18,22}$ As a result, Medicare has imposed financial penalties on hospitals with high readmission rates for specific conditions. ${ }^{19,23}$ Hospital-wide readmission rates are also currently published and presented to the public as a measure of hospital quality. ${ }^{1,26,28}$ While age, disease severity, and medical comorbidities have clearly been shown to contribute to readmission, the impact of specific clinical practices is less certain but likely plays a role in some instances..$^{19,24}$ For example, in acute ischemic stroke, readmission varies

ABBREVIATIONS $\mathrm{CCI}=$ Charlson Comorbidity Index; $\mathrm{CHF}=$ congestive heart failure; $\mathrm{GCS}=$ Glasgow Coma Scale; $\mathrm{GOS}=$ Glasgow Outcome Scale; ICU = intensive care unit; IQR = interquartile range; SDH = subdural hematoma.

SUBMITTED January 21, 2017. ACCEPTED June 19, 2017.

INCLUDE WHEN CITING Published online December 22, 2017; DOI: 10.3171/2017.6.JNS17188. 
considerably at the hospital level and is associated with specific practice patterns. ${ }^{4}$ Reducing unexpected readmission through improved discharge planning, medication reconciliation, and other means is an important current focus of hospital quality improvement efforts. ${ }^{8,19}$

Subdural hematoma (SDH) is a common disease, with more than 90,000 hospitalizations in the US, costing approximately $\$ 1.6$ billion, in 2007.9 The frequency of SDH hospitalizations has been increasing and is expected to continue to grow, in part due to an aging population. ${ }^{2,9,33}$ For elderly patients, chronic SDHs have been found to increase mortality and the diagnosis has been described as a sentinel health event similar to hip fractures. ${ }^{7,20}$ Despite their frequency and significance, to our knowledge the incidence and clinical predictors of all-cause 30-day readmission rates in patients with SDHs have not been described. We hypothesized that SDHs would be associated with a high rate of readmission due to deconditioning and medical comorbidities, as well as underlying disease severity.

Traditionally, SDH has been considered a surgical disease that is primarily managed via burr-hole drainage or craniotomy. Increasingly, however, conservative management without operative intervention is being used. $3,9,21,32$ The effect of this trend on patient outcomes is uncertain, and some publications have noted a higher mortality rate in conservatively managed patients. ${ }^{7,9}$ Based on this prior work and our clinical experience, we hypothesized that initial conservative management strategies would be associated with an increased frequency of unexpected, allcause, 30 -day readmission.

\section{Methods \\ Study Population}

This is a retrospective observational study of a cohort of 200 consecutive adult patients with SDH admitted to the University of Michigan neurosurgical intensive care unit (ICU) between March 2011 and July 2015. After approval was obtained from the University of Michigan institutional review board, patients were identified by searching billing records for ICD 9 codes 852.2 or 852.3 (traumatic $\mathrm{SDH}$ ) and 432.1 (nontraumatic SDH). These codes have previously been shown to have a $70 \%$ sensitivity, $100 \%$ specificity, $94 \%$ positive predictive value, and $100 \%$ negative predictive value for identifying SDH patients. ${ }^{9}$ The exclusion criteria were age less than 18 years; SDH due to a vascular cause, such as arteriovenous malformation or aneurysm; and any neurosurgical procedure within 30 days prior to the initial admission.

\section{Study Protocol and Parameters}

Using standardized collection forms and definitions, we abstracted information from the electronic medical record related to demographics, medical history, laboratory values, and standardized clinical scales, such as the Charlson Comorbidity Index (CCI $)^{5}$ and Glasgow Coma Scale (GCS). ${ }^{32}$ The GCS score was abstracted directly from entries made in the chart at the time of initial neurosurgical evaluation. Additionally, hematoma characteristics, surgical procedures, hospital complications and treatments, discharge disposition, presence of a 30-day readmission, and readmission features were identified. Initial conservative management of the SDH was defined as no surgical evacuation of the SDH during the index hospitalization. All data were stored in an online research electronic data capture (REDCap) database hosted at the University of Michigan. ${ }^{13}$

Maximum hematoma width and midline shift were measured on the worst preoperative head CT scan by a single investigator (C.A.W.). Hematoma appearance was classified as hyperdense, isodense/hypodense, or mixed density. Hyperdense hematomas were uniformly brighter than adjacent brain parenchyma, whereas isodense/hypodense hematomas had a Hounsfield unit value equal to or less than that of normal brain. Mixed-density hematomas contained both hyperdense and isodense or hypodense hematoma, with at least $1 \mathrm{CT}$ slice containing $20 \%$ hyperdense hematoma. ${ }^{14}$

\section{Determination of Outcome and Unexpected Readmission Status}

Outcome at hospital discharge was determined objectively by disposition, with a discharge to home or an acute rehabilitation hospital being considered a good outcome. Discharge to a skilled nursing facility, subacute rehabilitation hospital, or long-term acute care hospital was considered to be a poor outcome. The Glasgow Outcome Scale (GOS) score was also determined at the time of hospital discharge. ${ }^{16}$ The GOS score was obtained by review of the final neurological examination as well as final evaluations by physical and occupational therapy services. A good outcome was defined as a GOS score $>3$, which is defined as moderate disability or good recovery.

Readmitted patients discharged without documentation of a plan to return to the hospital for surgery or other treatments were considered to have had an unexpected readmission. Two investigators independently reviewed all readmissions and determined if the readmission was directly attributable to the SDH. Any differences were resolved by consensus. Examples of readmission reasons directly attributable to the SDH included new focal neurological deficits, hematoma expansion, surgical complications, headaches, seizures, or altered mental status not due to a toxic-metabolic etiology. Readmissions not directly attributable to the SDH were due to another medical condition (for example, urinary tract infection or venous thromboembolism), which was potentially exacerbated or triggered by recent illness and hospitalization.

\section{Determination of Treatment Costs}

Total facility charges for each admission and readmission were obtained by querying the hospital administrative database. Direct costs were then estimated by multiplying the total admissions charges by our facility's Medicare cost-to-charge ratio, obtained online from the Centers for Medicare and Medicaid Services (https:// www.cms.gov/Medicare/Medicare-Fee-for-ServicePayment/AcuteInpatientPPS/Historical-Impact-Files-forFY-1994-through-Present.html, accessed 4.4.2017). ${ }^{31} \mathrm{Be}-$ cause the data are strongly skewed to the right due to a small number of large values, all cost data are presented as median charge (interquartile range [IQR]). 


\section{Clinical Care}

Our institution is a level I trauma center. All patients with new SDH are initially admitted to an ICU to facilitate close monitoring for neurological deterioration. Patients with significant polytrauma are typically admitted to a dedicated trauma ICU, whereas those with isolated head trauma are admitted to the neurosurgical ICU, from which the population of this study is drawn. Following any neurosurgical intervention for $\mathrm{SDH}$, including craniotomy and burr-hole drainage, patients with isolated head trauma or SDH are admitted to the neurosurgical ICU. Our institution does not use the bedside subdural evacuating port system. Polytrauma patients who undergo a neurosurgical intervention are sometimes also admitted to the neurosurgical ICU for postoperative care. All decisions to intervene operatively and the specific types of procedures performed are at the discretion of the attending neurosurgeon.

\section{Statistical Analysis}

Demographic and clinical characteristics were compared between patients with and without unexpected 30day readmission. Categorical variables were evaluated using chi-square or Fisher's exact tests. Continuous variables were compared with t-tests or the Wilcoxon ranksum test. The association between surgical management and unexpected readmission was evaluated using inverse probability of treatment weighting (IPTW) by propensity scores. Propensity score methods are increasingly being used in observational studies of surgical interventions.
By determining the probability of receiving a procedure, propensity scores minimize the impact of confounding by indication. ${ }^{27}$ Inverse probability of treatment weighting is a specific type of propensity score method, in which covariate balance is achieved between treatment groups by assigning weights to subjects based on their individual probability of receiving their given treatment. In this way, the analyzed cohorts are more akin to the balanced cohorts achieved by randomization, permitting less-biased comparison between treatment groups. ${ }^{1,6}$

In this study, logistic regression was used to calculate propensity scores representing the predicted probability of surgery based on 10 potentially confounding covariates, which were selected based on their independent association with surgical management and SDH outcome, as well as review of the literature. The covariates were age, sex, GCS score, presence of focal neurological deficits, hematoma width, midline shift, antiplatelet medication use, history of congestive heart failure (CHF), history of stroke, and history of dementia. Propensity scores for surgically managed and conservatively managed patients were inspected for overlap, and 36 patients with scores outside the range of the other group were excluded from the analysis. ${ }^{30}$ The initial distribution of propensity scores, prior to exclusion of subjects with nonoverlapping scores, is shown in Fig. 1. Stabilized treatment weights were then calculated for each patient. ${ }^{12,25}$ Full details of the methods that were used to calculate propensity scores and stabilized treatment weights are available as Supplemental Materials.

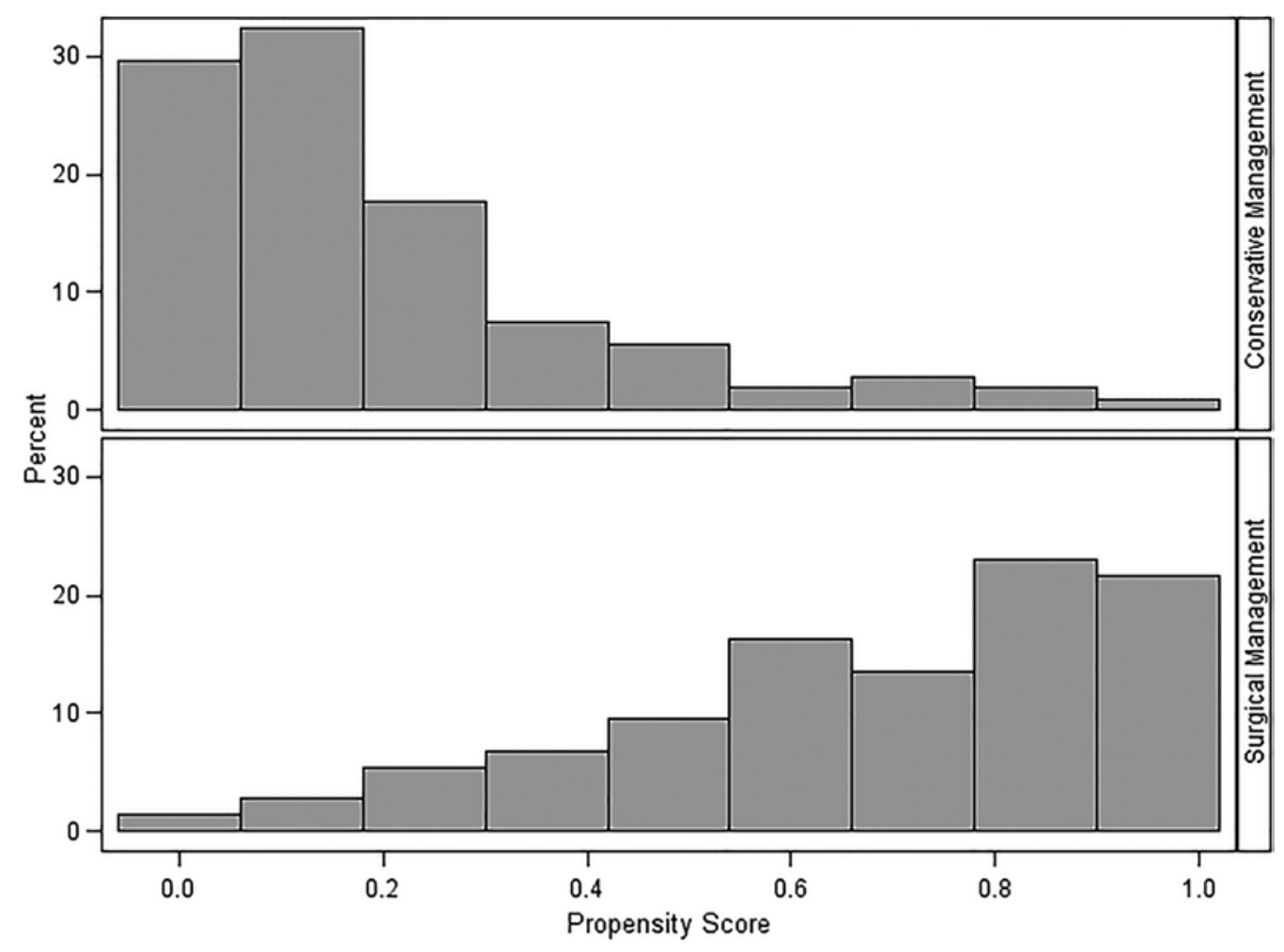

FIG. 1. Distribution of propensity scores in 74 surgically and 108 conservatively managed patients. The histograms display the distribution of propensity scores for the probability of surgical treatment for the conservative management and surgical management groups. 
The association between surgery and unexpected readmission was then evaluated by simple and multivariable logistic regression weighted by the stabilized inverse probability of treatment weights. Multivariable logistic regression included additional likely confounders, namely age, gender, GCS score, and hematoma width. Since these variables are all included in the propensity score model for surgical intervention, their inclusion in the weighted logistic regression model controls for additional confounding unrelated to surgical treatment decisions. In secondary analyses, the logistic regression models were repeated, with the outcome variable limited to unexpected readmissions that were directly attributable to the SDH and those that were not attributable to SDH. Finally, the same models with the outcome restricted to readmissions requiring surgical management were tested.

Because inverse probability of treatment analyses can be disproportionately influenced by large weights, as a sensitivity analysis the weighted logistic regression analysis was repeated with removal of outlying weights. The above analyses were also repeated using the full data set, without excluding patients with nonoverlapping propensity scores. Full details regarding the distribution of stabilized weights and sensitivity analysis are also available in the Supplemental Materials. Statistical significance was set at $\mathrm{p}<0.05$ (2-sided). All analyses were performed using SAS, version 9.4 (SAS Institute Inc.).

\section{Results}

\section{Description of the Population}

Of the 200 patients who met the inclusion criteria, 18 (9\%) died prior to discharge and were not included in the analysis. The mean age of the 182 included patients was 67.8 years; $115(63.2 \%)$ were male, and 74 patients $(40.7 \%)$ underwent surgery during their initial hospitalization. Of 50 total readmissions, 2 were for scheduled cranial surgeries. Consequently, 48 patients $(26 \%)$ were unexpectedly readmitted within 30 days of discharge.

\section{Univariate Analysis of Readmission}

Prehospitalization and admission patient characteristics are displayed in Table 1. Only sex was significantly associated with unexpected readmission, with $50 \%$ of readmitted patients being female versus $32.1 \%$ of those not readmitted $(\mathrm{p}=0.03)$. Age, comorbidities, presence of coagulopathies, and clinical and radiographic severity did not significantly differ between the 2 groups. Hospital and discharge characteristics are compared in Table 2 . Only conservative management was significantly associated with unexpected 30-day readmission. Of patients unexpectedly readmitted, 35 (72.9\%) were managed conservatively in comparison with $73(54.5 \%)$ of the patients not readmitted $(p=0.03)$. The type of surgery, discharge disposition, and discharge GOS score did not differ significantly between the 2 groups.

\section{Comparison of Surgically Versus Conservatively Managed Patients}

Of 182 patients surviving to initial hospital discharge, 74 (40.7\%) were treated surgically, while 108 (59.3\%) were
TABLE 1. Demographic and initial clinical characteristics

\begin{tabular}{lccc}
\hline \multicolumn{1}{c}{ Characteristic } & $\begin{array}{c}\text { Unexpectedly } \\
\text { Readmitted } \\
(\mathrm{n}=48)\end{array}$ & $\begin{array}{c}\text { Not Unexpectedly } \\
\text { Readmitted } \\
(\mathrm{n}=134)\end{array}$ & $\begin{array}{c}\mathrm{p} \\
\text { Value }\end{array}$ \\
\hline Mean age in yrs & $69.1(16.4)$ & $67.4(16.4)$ & 0.54 \\
\hline Female sex & $24(50.0)$ & $43(32.1)$ & 0.03 \\
\hline Mean CCI & $2.3(2.2)$ & $2.0(2.1)$ & 0.39 \\
\hline Dementia & $6(12.5)$ & $22(16.4)$ & 0.52 \\
\hline CAD & $10(20.8)$ & $33(24.6)$ & 0.60 \\
\hline CHF & $5(10.4)$ & $19(14.2)$ & 0.51 \\
\hline Prior CVA & $9(18.8)$ & $24(17.9)$ & 0.90 \\
\hline Thrombocytopenia & $8(16.7)$ & $10(7.5)$ & 0.09 \\
\hline Taking antiplatelet drugs & $18(37.5)$ & $62(46.3)$ & 0.29 \\
\hline Taking anticoagulants & $12(25.0)$ & $30(22.4)$ & 0.71 \\
\hline Antecedent trauma & $35(72.9)$ & $98(73.1)$ & 0.98 \\
\hline Mean GCS score & $14.3(1.7)$ & $13.6(2.6)$ & 0.18 \\
\hline Focal neuro deficits & $11(22.9)$ & $46(34.3)$ & 0.14 \\
\hline Hematoma characteristics & & & 0.79 \\
\hline \multicolumn{1}{c}{ Hyperdense } & $21(43.8)$ & $65(48.5)$ & \\
\hline Iso-/hypodense & $6(12.5)$ & $18(13.4)$ & \\
\hline \multicolumn{1}{c}{ Mixed } & $21(43.8)$ & $51(38.1)$ & \\
\hline Mean max hematoma & $12.4(6.6)$ & $13.1(7.2)$ & 0.55 \\
\hline width in mm & & & 0.62 \\
\hline Mean midline shift in mm & $3.8(3.7)$ & $4.2(4.3)$ & \\
\hline $\begin{array}{l}\text { CAD = coronary artery disease; CCI = Charlson Comorbidity Index; CVA } \\
\text { cerebrovascular accident; neuro }\end{array}$ & neurological. & & \\
$\begin{array}{l}\text { Values are number of cases }(\%) \\
\text { with SDs. }\end{array}$ & & & \\
\hline
\end{tabular}

managed conservatively. A comparison of initial clinical characteristics of patients who were managed surgically versus those managed conservatively is shown in Table 3. Initial characteristics are highlighted here, as these are the features that influence surgical decision making. As would be expected, surgically managed patients were significantly more likely to have focal neurological deficits $(48.7 \%$ vs $19.5 \%, \mathrm{p}<0.0001)$ and had significantly greater

TABLE 2. Treatment and discharge characteristics

\begin{tabular}{lccc}
\hline \multicolumn{1}{c}{ Characteristic } & $\begin{array}{c}\text { Unexpectedly } \\
\text { Readmitted } \\
(\mathrm{n}=48)\end{array}$ & $\begin{array}{c}\text { Not Unexpectedly } \\
\text { Readmitted } \\
(\mathrm{n}=134)\end{array}$ & $\begin{array}{c}\mathrm{p} \\
\text { Value }\end{array}$ \\
\hline Conservative management & $35(72.9)$ & $73(54.5)$ & 0.03 \\
\hline Surgery type & & & 0.71 \\
\hline Burr-hole drainage & $8(16.7)$ & $37(27.6)$ & \\
\hline Craniotomy & $5(10.4)$ & $19(14.2)$ & \\
\hline Craniectomy & $0(0.0)$ & $5(3.7)$ & \\
\hline Good discharge outcome* & $24(50)$ & $58(43.3)$ & 0.42 \\
\hline $\begin{array}{l}\text { Discharge to home or } \\
\text { acute rehab }\end{array}$ & $32(66.7)$ & $96(71.6)$ & 0.52 \\
\hline
\end{tabular}

Rehab = rehabilitation.

Values are number of cases (\%).

* GOS score of 4 or 5 . 
TABLE 3. Covariates of interest in weighted and unweighted samples

\begin{tabular}{|c|c|c|c|c|c|c|}
\hline \multirow[b]{2}{*}{ Clinical Covariates } & \multicolumn{3}{|c|}{ Unweighted Sample } & \multicolumn{3}{|c|}{ Weighted Sample } \\
\hline & $\begin{array}{l}\text { Conservative Management } \\
\qquad(n=108)\end{array}$ & $\begin{array}{l}\text { Surgical Management } \\
\qquad(n=74)\end{array}$ & $\begin{array}{c}p \\
\text { Value }\end{array}$ & $\begin{array}{l}\text { Conservative Management } \\
\qquad(n=79)\end{array}$ & $\begin{array}{l}\text { Surgical Management } \\
\qquad(\mathrm{n}=67)\end{array}$ & $\begin{array}{c}p \\
\text { Value }\end{array}$ \\
\hline Age & $69.5(16.7)$ & $65.4(15.8)$ & 0.11 & $65.4(20.5)$ & $67.9(13.4)$ & 0.40 \\
\hline Male sex & 55.6 & 74.3 & 0.01 & 69.9 & 57.8 & 0.14 \\
\hline Focal neuro deficits & 19.4 & 48.7 & $<0.001$ & 33.6 & 34.5 & 0.91 \\
\hline Hematoma width & $9.4(5.6)$ & $18.1(5.8)$ & $<0.001$ & $14.6(7.8)$ & $14.8(5.3)$ & 0.80 \\
\hline Midline shift & $2.0(2.4)$ & $7.1(4.3)$ & $<0.001$ & $3.4(2.7)$ & $5.3(3.7)$ & 0.002 \\
\hline GCS score & $14.0(1.9)$ & $13.4(2.9)$ & 0.53 & $14.6(7.8)$ & $13.6(2.5)$ & 0.15 \\
\hline $\mathrm{CCl}$ score & $2.2(2.2)$ & $1.9(2.1)$ & 0.38 & $1.8(2.3)$ & $2.2(2.0)$ & 0.33 \\
\hline Thrombocytopenia & 11.1 & 8.1 & 0.5 & 13.7 & 6.1 & 0.15 \\
\hline INR $>2$ & 18.5 & 16.2 & 0.69 & 14.9 & 13.3 & 0.78 \\
\hline Antiplatelet meds & 51.9 & 32.4 & 0.01 & 32.2 & 33.1 & 0.92 \\
\hline
\end{tabular}

$\mathrm{CCl}$ = Charlson Comorbidity Index; INR = international normalized ratio; meds = medications.

Values are percentages for categorical variables and mean (SD) for continuous variables.

hematoma width (18.1 vs $9.4 \mathrm{~mm}, \mathrm{p}<0.001)$ and midline shift (7.1 vs $2.0 \mathrm{~mm}, \mathrm{p}<0.001)$. They were also significantly less likely to have a diagnosis of CHF or dementia and to be taking antiplatelet medications at the time of admission. Among 74 surgically managed patients, 13 $(17.6 \%)$ were unexpectedly readmitted, as compared with $35(32.4 \%)$ of 108 conservatively managed patients $(\mathrm{p}=$ $0.03)$.

Overall, surgically managed patients had a significantly greater hospital length of stay than conservatively managed patients (mean 10.2 vs 5.6 days, $\mathrm{p}<0.001$ ). Outcomes at the time of hospital discharge did not differ significantly between the 2 groups. In the conservatively managed group, $50 \%$ of the patients had a GOS score $>3$ at hospital discharge, as compared with $37.8 \%$ of surgically treated patients $(p=0.10)$. The percentage of patients well enough to be discharged to home or acute rehabilitation was $67.6 \%$ in the conservatively managed group and $74.3 \%$ in those treated surgically $(\mathrm{p}=0.33)$.

\section{Readmission Characteristics}

The median time to readmission was 6.0 days (IQR 3-11 days), with 30 (63\%) patients being readmitted at or prior to 7 days (Fig. 2). Of 48 patients with unexpected 30day readmissions, 24 patients (13\%) were readmitted for reasons directly attributable to the $\mathrm{SDH}$, most commonly headaches, followed by new focal neurological deficits. Of these 24, 19 (79\%) were managed conservatively versus 89 $(56 \%)$ of the 158 patients who were not readmitted within 30 days for reasons directly attributable to the SDH ( $\mathrm{p}=$ $0.03)$. The other 24 unexpected readmissions (13\%) were readmitted for reasons that were determined to not be directly attributable to the SDH. The most common reason for readmission not directly due to the SDH was an exacerbation of a preexisting medical condition, such as malignancy or hypertensive urgency. Dehydration and electrolyte abnormalities were also quite common, followed by venous thromboembolism.

Patients readmitted for reasons other than SDH did not significantly differ in the proportion receiving conserva- tive management. Sixteen (67\%) of 24 were managed conservatively, whereas $58 \%$ of the other 158 patients were managed conservatively $(\mathrm{p}=0.43)$.

Of the 48 unexpectedly readmitted patients, 16 (33.3\%) required surgical intervention during their readmission. Of these, $14(87.5 \%)$ were managed conservatively during their initial hospitalization. Overall, patients managed conservatively during their initial hospitalization were significantly more likely to be readmitted and require surgery $(p=0.02)$.

\section{Admission Costs}

The median direct hospital cost for initial SDH admission was \$20,355 (IQR \$12,672-\$36,733). The cost was less for patients whose cases were initially managed conservatively, with a median of $\$ 15,348$ (IQR $\$ 9070$ $\$ 22,519$ ) versus $\$ 31,211$ (IQR $\$ 31,211-\$ 56,004$ ) for surgically managed patients. The median cost for an unexpected readmission was $\$ 14,564$ (IQR \$6884-\$23,325). The median cost for readmission due to the SDH was higher, at \$17,908 (IQR \$9401-\$23,325), than that for readmission not directly attributable to the SDH, \$8982 (IQR \$6048$\$ 22,873$ ), but these results were not statistically significant $(\mathrm{p}=0.19)$. However, the median readmission cost was significantly higher when a surgery was performed during readmission: \$21,884 (IQR \$17,793-\$29,920) versus \$8982 (IQR \$5231-\$18,966) (p=0.004). Overall, in comparison with patients who undergo initial surgical management, the median additional direct cost for a patient with initial conservative management who required surgery upon readmission was $\$ 6021$.

\section{Propensity Score Analysis}

After treatment weights were applied, key covariates were much more balanced between groups, as shown in Table 3. The proportions of patients with focal neurological deficits and hematoma width, which had differed highly between the surgical and conservative groups $(\mathrm{p}<0.001$ for both), were virtually identical in the 2 groups when 


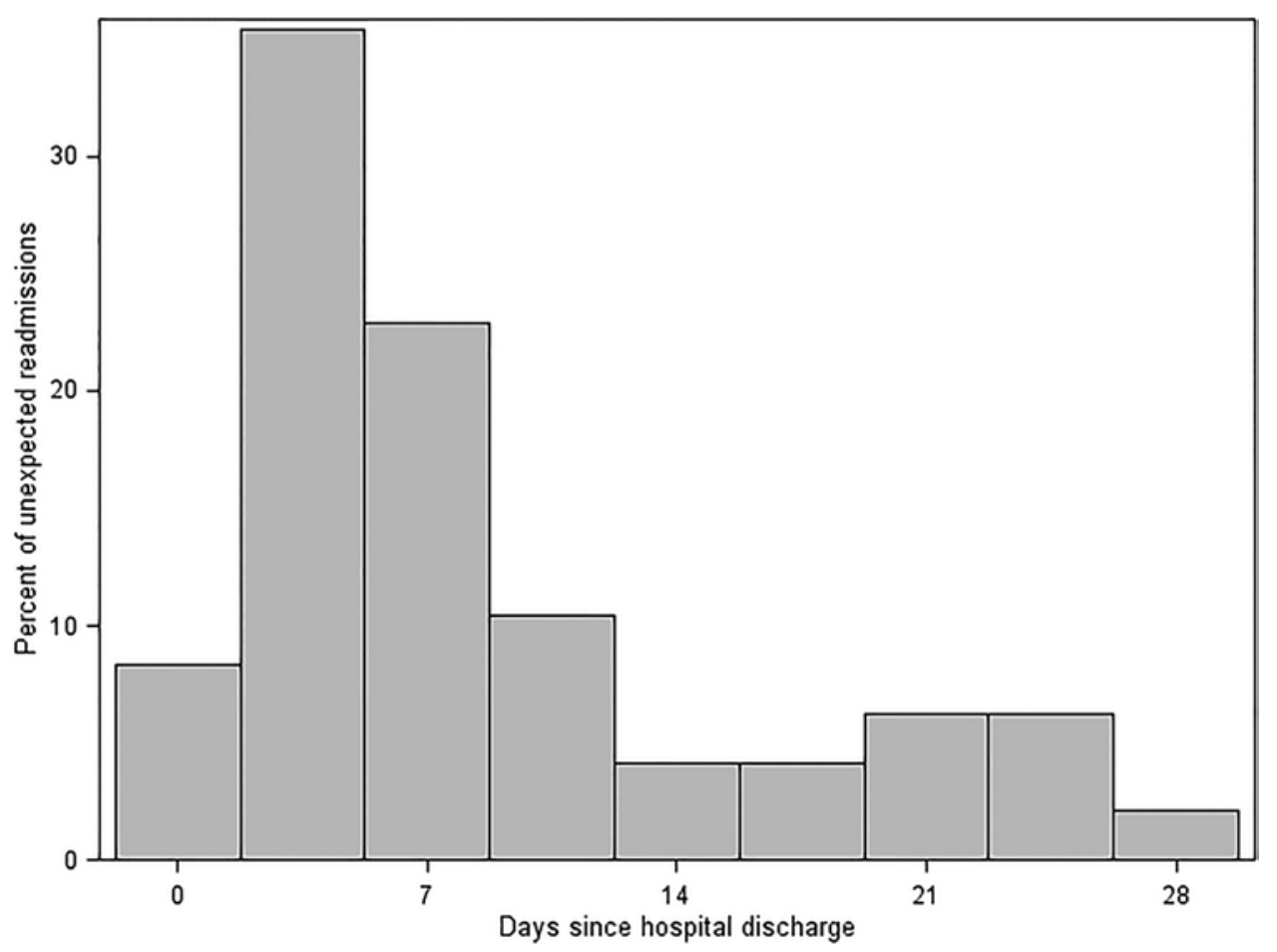

FIG. 2. Distribution of the time to unexpected readmission following hospital discharge. The histogram displays the timing of when patients were unexpectedly readmitted, in terms of the number of days since hospital discharge. The median number of days between discharge and unexpected readmission is 6.0 (IQR 3-11).

weights were applied. The difference in midline shift was reduced from 5.1 to $1.9 \mathrm{~mm}$, but this difference remained statistically significant $(\mathrm{p}=0.002)$.

In weighted logistic regression analysis, initial surgical intervention was not significantly associated with increased odds of a favorable outcome at hospital discharge in either the simple (OR 1.24, 95\% CI 0.54-2.83) or multivariable (OR 1.82, 95\% CI 0.65-5.09) models (Table 4). Increasing age was significantly associated with lower odds of favorable discharge outcome, whereas higher GCS score was significantly associated with favorable outcome. In contrast, surgical intervention was highly associated with lower odds of unexpected 30-day readmission in simple (OR $0.20,95 \%$ CI $0.08-0.47)$ and multivariable (OR $0.19,95 \%$ CI $0.08-0.49$ ) logistic regression analysis (Table 5). Hematoma width was also strongly associated with unexpected readmission (OR 1.12, 95\% CI 1.04-1.20), and age showed a statistically significant association. When the outcome was restricted to patients whose readmission was directly attributable to SDH, the strength of the association between surgery and decreased odds of readmission increased (OR 0.08, 95\% CI 0.02-0.34). In contrast, for patients readmitted for reasons not directly due to the SDH, the strength of the association decreased markedly and was no longer statistically significant (OR $0.89,95 \%$ CI 0.29 2.77). Similar results were obtained when the outcome was restricted to readmissions requiring surgical intervention. Initial surgical intervention was strongly associated with decreased odds of readmission requiring surgery in simple (OR 0.06, 95\% CI 0.01-0.37) and multivariable (OR 0.03, 95\% CI 0.004-0.23) weighted logistic regression analysis.
The full results of these analyses are available as Supplemental Materials in Tables S2-S4. In additional sensitivity analyses, the associations were largely unchanged. For example, with inclusion of the 36 subjects who were initially removed due to nonoverlapping propensity scores, the simple (OR $0.21,95 \%$ CI $0.09-0.49$ ) and multivariable (OR $0.16,95 \%$ CI $0.07-0.40$ ) associations were nearly the same. The full results of these sensitivity analyses are available in Supplemental Tables S5 and S6.

\section{Discussion}

In this study, we found that a substantial proportion of patients $(26.4 \%)$ admitted for SDH to a neurosurgical ICU at a single academic institution were unexpectedly readmitted within 30 days. Initial surgical intervention was not associated with outcome at hospital discharge, but there

TABLE 4. Weighted logistic regression analysis of favorable discharge disposition

\begin{tabular}{|c|c|c|c|c|c|c|}
\hline \multirow[b]{2}{*}{ Covariates } & \multicolumn{3}{|c|}{ Univariate Analysis } & \multicolumn{3}{|c|}{ Multivariable Model } \\
\hline & OR & $95 \% \mathrm{Cl}$ & $p$ Value & OR & $95 \% \mathrm{Cl}$ & $p$ Value \\
\hline Surgery & 1.24 & $0.54-2.83$ & 0.61 & 1.82 & $0.65-5.09$ & 0.25 \\
\hline Age & & & & 0.94 & $0.90-0.98$ & 0.002 \\
\hline Male sex & & & & 1.14 & $0.39-3.35$ & 0.98 \\
\hline $\begin{array}{l}\text { Hematoma } \\
\text { width }\end{array}$ & & & & 0.95 & $0.88-1.02$ & 0.16 \\
\hline GCS score & & & & 1.71 & $1.35-2.2$ & $<0.001$ \\
\hline
\end{tabular}


TABLE 5. Weighted logistic regression analysis of unexpected admission

\begin{tabular}{lccccccc}
\hline & \multicolumn{3}{c}{ Univariate Analysis } & & \multicolumn{3}{c}{ Multivariable Model } \\
\cline { 2 - 3 } \cline { 5 - 7 } Covariates & OR & $95 \% \mathrm{Cl}$ & $\mathrm{p}$ Value & & OR & $95 \% \mathrm{Cl}$ & $\mathrm{p} \mathrm{Value}$ \\
\hline Surgery & 0.20 & $0.08-0.47$ & 0.0003 & & 0.19 & $0.08-0.49$ & 0.0005 \\
\hline Age & & & & 0.97 & $0.95-1.00$ & 0.02 \\
\hline Male sex & & & 0.99 & $0.40-2.45$ & 0.98 \\
\hline $\begin{array}{l}\text { Hematoma } \\
\text { width }\end{array}$ & & 1.12 & $1.04-1.20$ & 0.0009 \\
\hline GCS score & & & 1.17 & $0.89-1.54$ & 0.25 \\
\hline
\end{tabular}

was a statistically significant association between initial conservative management and unexpected 30-day readmission. This association remained significant after adjustment for the likelihood of surgical intervention with logistic regression weighted by the inverse probability of treatment using propensity scores. The odds ratio of 0.20 in simple inverse probability of treatment weighted logistic regression analysis implies that, for patients with a similar likelihood of receiving surgical intervention, conservative management is associated with 5-fold greater odds of unexpected readmission.

Female sex was the only other factor that was significantly associated with unexpected 30-day readmission in univariate analysis. However, in weighted multivariable logistic regression analysis, this association was no longer statistically significant. It is somewhat surprising that other clinical factors, such as age, GCS score, presence of neurological deficits, and radiographic severity of $\mathrm{SDH}$, were not associated with increased likelihood of unexpected readmission. This may in part be due to the tendency of patients with greater clinical severity to undergo initial operative management, which appears to be protective against readmission. The majority of unexpected readmissions occurred within 7 days of discharge, suggesting that targeted interventions at, or shortly after, discharge could reduce unexpected readmission in this population. Costs were higher among patients receiving initial conservative management who then required surgery during their readmission. However, the median increase in direct costs was relatively modest, at $\$ 6021$. Due to the retrospective nature of this study, the impact on long-term costs and patient outcomes could not be ascertained.

This study's finding of significantly reduced odds of readmission with surgical management is novel, and there are no prior studies for direct comparison. However, this finding is concordant with previous observational studies finding improved outcomes in surgically treated patients. For example, surgical intervention for SDHs is associated with a reduction in mortality in select patients. ${ }^{7,917}$ Overall, $16(33.3 \%)$ of 48 unexpectedly readmitted patients required surgery during their readmission. Only $2(12.5 \%)$ of these 16 patients were treated surgically during their index hospitalization. In subgroup analysis, surgery was more strongly associated with decreased odds of readmission in patients whose readmission was directly attributable to the SDH. However, for patients who were readmitted for another condition, surgery was not associated with decreased odds of readmission. This further suggests that conserva- tive management was a significant contributor in many readmissions, but also illustrates that multiple other factors influence this outcome. Additionally, although costs were higher in the subgroup of patients who required surgical management during their readmission, the absolute cost increase was relatively modest.

To our knowledge, all-cause readmission rates following hospitalization for SDH have not previously been described. One study performed by Morris et al. ${ }^{21}$ observed an 11.8\% 90-day, SDH-related readmission rate in patients initially managed conservatively. Dumont et al. ${ }^{7}$ found an 8\% SDH-related readmission rate for elderly patients with chronic SDHs over a varying time span. In our study, the percentage of patients with readmissions directly attributable to SDHs was similar, at $13 \%$ within 30 days. Among Medicare recipients, the 30-day readmission rate has been reported to be approximately $20 \% .{ }^{15}$ The rate of $26.4 \%$ in this study is higher and closer to that of patients hospitalized with CHF or chronic obstructive pulmonary disease (COPD). ${ }^{29}$ This high rate may, in part, reflect an overall greater severity of illness among this study's population of patients admitted to a neurosurgical ICU at an academic referral center.

There are a number of important limitations of this study that should be noted. The study population was derived from a single neurosurgical ICU, so generalization of the results to other ICUs and institutions is uncertain and should be examined in follow-up studies. Similarly, electronic medical record data detailing readmissions at other institutions were not available to the study team, so it is likely that some patients who were readmitted to other institutions were not appropriately identified. Accurate recognition of some clinical covariates and outcomes may have also been affected by the study's retrospective nature. Additionally, we were not able to accurately determine long-term costs and clinical outcomes for surgically and conservatively managed patients.

Although we attempted to account for confounding by indication for surgery through the determination of propensity scores and the use of inverse probability of treatment weighted logistic regression, we cannot rule out the influence of unmeasured confounders as well as incomplete adjustment for known confounders. As shown in Table 3, trimming and weighting of the sample resulted in a study population with covariates that are much more balanced between conservatively and surgically managed groups. However, some residual differences did remain and may have influenced the study results. The one remaining statistically significant difference was a $1.9 \mathrm{~mm}$ greater midline shift in the surgically managed cohort. If anything, however, this greater illness severity would be expected to bias the surgical group toward a higher readmission rate, which was not observed. In the absence of a clinical trial, research investigating the impact of conservative management on readmission following SDH hospitalizations will also depend on observational data that are subject to similar limitations.

\section{Conclusions}

The burden of unexpected 30-day readmissions following hospitalization at an academic neurosurgical ICU for 
SDH is substantial. Initial surgical management, in comparison with conservative management, is strongly associated with decreased odds of unexpected readmission, even when controlling for likely confounders in a multivariable logistic regression model weighted by the inverse probability of treatment using propensity scores. However, surgical intervention did not impact patient outcome at the time of hospital discharge. Additionally, the total cost increase for patients who undergo initial conservative management and then are readmitted for surgery is relatively modest. Future studies should seek to evaluate the impact of conservative management on long-term costs and clinical outcomes in a larger population, ideally from multiple centers. Ultimately, a clinical trial is needed to fully evaluate the impact of conservative management in patients with SDH.

\section{Acknowledgments}

Craig Williamson received funding from the Massey Foundation TBI Grand Challenge Grant to support a portion of this study. Lynze Franko received funding from the University of Michigan School of Medicine for conference attendance to present portions of this work.

\section{References}

1. Austin PC, Stuart EA: Moving towards best practice when using inverse probability of treatment weighting (IPTW) using the propensity score to estimate causal treatment effects in observational studies. Stat Med 34:3661-3679, 2015

2. Balser D, Farooq S, Mehmood T, Reyes M, Samadani U: Actual and projected incidence rates for chronic subdural hematomas in United States Veterans Administration and civilian populations. J Neurosurg 123:1209-1215, 2015

3. Bullock MR, Chesnut R, Ghajar J, Gordon D, Hartl R, Newell DW, et al: Surgical management of acute subdural hematomas. Neurosurgery 58 (3 Suppl):S16-S24, Si-Siv, 2006

4. Burke JF, Skolarus LE, Adelman EE, Reeves MJ, Brown DL: Influence of hospital-level practices on readmission after ischemic stroke. Neurology 82:2196-2204, 2014

5. Charlson ME, Pompei P, Ales KL, MacKenzie CR: A new method of classifying prognostic comorbidity in longitudinal studies: development and validation. J Chronic Dis 40:373383, 1987

6. Curtis LH, Hammill BG, Eisenstein EL, Kramer JM, Anstrom KJ: Using inverse probability-weighted estimators in comparative effectiveness analyses with observational databases. Med Care 45 (10 Suppl 2):S103-S107, 2007

7. Dumont TM, Rughani AI, Goeckes T, Tranmer BI: Chronic subdural hematoma: a sentinel health event. World Neurosurg 80:889-892, 2013

8. Field TS, Ogarek J, Garber L, Reed G, Gurwitz JH: Association of early post-discharge follow-up by a primary care physician and 30-day rehospitalization among older adults. J Gen Intern Med 30:565-571, 2015

9. Frontera JA, Egorova N, Moskowitz AJ: National trend in prevalence, cost, and discharge disposition after subdural hematoma from 1998-2007. Crit Care Med 39:1619-1625, 2011

10. Gonzalez AA, Abdelsattar ZM, Dimick JB, Dev S, Birkmeyer JD, Ghaferi AA: Time-to-readmission and mortality after high-risk surgery. Ann Surg 262:53-59, 2015

11. Greenblatt DY, Weber SM, O'Connor ES, LoConte NK, Liou JI, Smith MA: Readmission after colectomy for cancer predicts one-year mortality. Ann Surg 251:659-669, 2010

12. Harder VS, Stuart EA, Anthony JC: Propensity score techniques and the assessment of measured covariate balance to test causal associations in psychological research. Psychol Methods 15:234-249, 2010

13. Harris PA, Taylor R, Thielke R, Payne J, Gonzalez N, Conde JG: Research electronic data capture (REDCap) - a metadata-driven methodology and workflow process for providing translational research informatics support. J Biomed Inform 42:377-381, 2009

14. Honda Y, Sorimachi T, Momose H, Takizawa K, Inokuchi S, Matsumae M: Chronic subdural haematoma associated with disturbance of consciousness: significance of acute-onchronic subdural haematoma. Neurol Res 37:985-992, 2015

15. Jencks SF, Williams MV, Coleman EA: Rehospitalizations among patients in the Medicare fee-for-service program. $\mathbf{N}$ Engl J Med 360:1418-1428, 2009

16. Jennett B, Bond M: Assessment of outcome after severe brain damage: a practical scale. Lancet 1:480-484, 1975

17. Lee L, Ker J, Ng HY, Munusamy T, King NK, Kumar D, et al: Outcomes of chronic subdural hematoma drainage in nonagenarians and centenarians: a multicenter study. J Neurosurg 124:546-551, 2016

18. Lum HD, Studenski SA, Degenholtz HB, Hardy SE: Early hospital readmission is a predictor of one-year mortality in community-dwelling older Medicare beneficiaries. J Gen Intern Med 27:1467-1474, 2012

19. McIlvennan CK, Eapen ZJ, Allen LA: Hospital readmissions reduction program. Circulation 131:1796-1803, 2015

20. Miranda LB, Braxton E, Hobbs J, Quigley MR: Chronic subdural hematoma in the elderly: not a benign disease. J Neurosurg 114:72-76, 2011

21. Morris NA, Merkler AE, Parker WE, Claassen J, Connolly ES, Sheth KN, et al: Adverse outcomes after initial non-surgical management of subdural hematoma: a population-based study. Neurocrit Care 24:226-232, 2016

22. Nuño M, Ly D, Mukherjee D, Ortega A, Black KL, Patil CG: Quality of surgical care and readmission in elderly glioblastoma patients. Neurooncol Pract 1:33-39, 2014

23. Patient Protection and Affordable Care Act of 2010, Pub Law 111-148, 124 Stat. 127, Sec. 6301 (March 23, 2010) (http:// www.gpo.gov/fdsys/pkg/PLAW-111publ148/pdf/PLAW111publ148.pdf)

24. Pollock FH, Bethea A, Samanta D, Modak A, Maurer JP, Chumbe JT: Readmission within 30 days of discharge after hip fracture care. Orthopedics 38:e7-e13, 2015

25. Robins JM, Hernán MA, Brumback B: Marginal structural models and causal inference in epidemiology. Epidemiology 11:550-560, 2000

26. Rosen AK, Chen Q, Shwartz M, Pilver C, Mull HJ, Itani KFM, et al: Does use of a hospital-wide readmission measure versus condition-specific readmission measures make a difference for hospital profiling and payment penalties? Med Care 54:155-161, 2016

27. Rosenbaum PR, Rubin DB: The central role of the propensity score in observational studies for causal effects. Biometrika 70:41-55, 1983

28. Schwartz J, Strait KM, Keshawarz A, Vellanky SS, Reilly EM, Curtis JP, et al: Medicare Hospital Quality Chartbook. Performance Report on Outcome Measures. September 2014. (https://www.cms.gov/medicare/ quality-initiatives-patient-assessment-instruments/ hospitalqualityinits/downloads/medicare-hospital-qualitychartbook-2014.pdf) [Accessed August 28, 2017]

29. Sharma G, Kuo YF, Freeman JL, Zhang DD, Goodwin JS: Outpatient follow-up visit and 30-day emergency department visit and readmission in patients hospitalized for chronic obstructive pulmonary disease. Arch Intern Med 170:16641670,2010

30. Stürmer T, Joshi M, Glynn RJ, Avorn J, Rothman KJ, Schneeweiss S: A review of the application of propensity score methods yielded increasing use, advantages in specific 
settings, but not substantially different estimates compared with conventional multivariable methods. J Clin Epidemiol 59:437-447, 2006

31. Taira DA, Seto TB, Siegrist R, Cosgrove R, Berezin R, Cohen DJ: Comparison of analytic approaches for the economic evaluation of new technologies alongside multicenter clinical trials. Am Heart J 145:452-458, 2003

32. Teasdale G, Jennett B: Assessment and prognosis of coma after head injury. Acta Neurochir (Wien) 34:45-55, 1976

33. Wiener JM, Tilly J: Population ageing in the United States of America: implications for public programmes. Int J Epidemiol 31:776-781, 2002

34. Wong CW: Criteria for conservative treatment of supratentorial acute subdural haematomas. Acta Neurochir (Wien) 135:38-43, 1995

\section{Disclosures}

The authors report no conflict of interest concerning the materials or methods used in this study or the findings specified in this paper.

\section{Author Contributions}

Conception and design: Williamson, Franko, Sheehan, Roark,
Joseph, Rajajee. Acquisition of data: Williamson, Franko, Joseph. Analysis and interpretation of data: Williamson, Franko, Burke, Rajajee. Drafting the article: Franko. Critically revising the article: Williamson, Sheehan, Roark, Joseph, Burke, Rajajee. Reviewed submitted version of manuscript: all authors. Approved the final version of the manuscript on behalf of all authors: Williamson. Statistical analysis: Williamson, Burke.

\section{Supplemental Information}

\section{Online-Only Content}

Supplemental material is available with the online version of the article.

Supplemental Materials. https://thejns.org/doi/suppl/10.3171/ 2017.6.JNS17188.

\section{Previous Presentations}

Portions of this work were presented as an oral platform presentation at the 2016 Neurocritical Care Society annual meeting in National Harbor, Maryland, on September 16, 2016.

\section{Correspondence}

Craig Williamson, Department of Neurosurgery, University of Michigan, 1500 E Medical Center Dr., Ann Arbor, MI 48109. email: craigaw@med.umich.edu. 